\title{
PARKOUR: MENSURAÇÃO DO METABOLISMO ENERGÉTICO MORFOFISIOLÓGICO DE SEUS PRATICANTES
}

\author{
PARKOUR: MEASUREMENT OF ENERGETIC AND MORPHOPHISIOLOGICAL \\ METABOLISM IN ITS PRACTITIONERS
}

Artigo Original

Original ARTICLE

Artículo Original

\author{
PARKOUR: MEDICIÓN DEL METABOLISMO ENERGÉTICO Y MORFOFISIOLÓGICO \\ DE SUS PRACTICANTES
}

\author{
Cassio Dias de Andrade Júnior ${ }^{1,2}$ \\ (Educador Físico) \\ Guilherme Natan Paiano dos Santos' \\ (Educador Físico) \\ Ana Cláudia Ferreira ${ }^{1}$ \\ (Educadora Física) \\ Marcelo Romanovitch Ribas ${ }^{1,2}$ \\ (Educador Físico) \\ Julio Cesar Bassan² \\ (Educador Físico) \\ 1. Faculdade Dom Bosco, Curso de \\ Educação Física, Curitiba, PR, Brasil. \\ 2. Universidade Tecnológica Federal \\ do Paraná, Curitiba, PR, Brasil.
}

\section{Correspondência:}

Avenida São José, 500. Apto. 64. Cristo Rei, Curitiba, PR, Brasil. 80050-350

juniorcassio1004@gmail.com

\section{RESUMO}

Introdução: O parkour é uma atividade que utiliza o próprio corpo para deslocar-se de um ponto a outro no ambiente. Objetivo: Analisar o comportamento do metabolismo energético e do sistema cardiorrespiratório durante uma sessão de parkour. Método: Foram avaliados 18 indivíduos praticantes da modalidade. O comportamento do metabolismo energético durante uma sessão de parkour foi avaliado por meio da frequência cardíaca (FC), lactato e glicose. Resultados: Os resultados indicaram que a FC média durante a sessão de treino foi de 167,64 7,05 bpm representando $84,36 \pm 3,52 \%$. O lactato inicial foi de $4,49 \pm 3,08 \mathrm{mmol} / \mathrm{L}$ e o final de $9,23 \pm 3,08 \mathrm{mmol} / \mathrm{L}$ onde $\mathrm{p}$-valor para as variáveis foi de $\mathrm{p}=0,0007$. A glicose inicial foi de $73,6 \pm 20,71 \mathrm{mg} / \mathrm{dL}$ e final de $76,06 \pm 20,20 \mathrm{mg} / \mathrm{dL}$ assumindo um $p$-valor de $p=0,7408$. Em se tratando da composição corporal, o percentual de gordura foi de 8,78 $\pm 2,82 \%$, a dobra que mais apresentou acúmulo de gordura foi do abdômen 12,32 \pm 5,69 mm. Conclusão: Conclui-se que a modalidade parkour tem predominância do metabolismo anaeróbio glicolítico, com suporte do metabolismo aeróbio. Além disso, devido à especificidade da modalidade, seus praticantes possuem baixo percentual de gordura.

Descritores: exercício; análise química do sangue; metabolismo energético.

\section{ABSTRACT}

Introduction: Parkour is an activity that uses the own body to move from a point to another in the environment. Objective: Analyzing the behavior of the energetic metabolism and the cardiorespiratory system during a parkour session. Method: Eighteen parkour practitioners were evaluated. The behavior of the energetic metabolism during the parkour session was measured by heart rate (HR), lactate and glucose. Results: The results indicated that the average HR during the training session was $167.64 \pm 7.05$ bpm representing $84.36 \pm 3.52 \%$. The initial lactate level was $4.49 \pm$ $3.08 \mathrm{mmol} / \mathrm{L}$ and the final level was $9.23 \pm 3.08 \mathrm{mmol} / \mathrm{L}$ where $p$-value for both variables was $p=0.0007$. The initial glucose was $73.6 \pm 20.71 \mathrm{mg} / \mathrm{dL}$ and the final glucose $76.06 \pm 20.20 \mathrm{mg} / \mathrm{dL}$, with a $p$-value of $p=0.7408$. In terms of body composition, the fat percentage was $8.78 \pm 2.82 \%$, the skinfold with highest fat amount was the abdomen $12.32 \pm 5.69 \mathrm{~mm}$. Conclusion: The conclusion was that the parkour modality has predominance of the anaerobic glycolytic metabolism, with support of the aerobic metabolism. Besides, due to the specificity of the modality, its practitioners have low levels of body fat.

Keywords: exercise; blood chemical analysis; energy metabolism.

\section{RESUMEN}

Introducción: Parkour es una actividad que utiliza el propio cuerpo para desplazarse de un punto a otro en el ambiente. Objetivo: Analizar el comportamiento del metabolismo energético y del sistema cardiorrespiratorio durante una sesión de parkour. Metodología: Se evaluaron 18 individuos que practican el la modalidadparkour. Fue evaluado el comportamiento del metabolismo energético durante una sesión de parkour a través de la frecuencia cardiaca (FC), lactato y glucosa. Resultados: Los resultados indicaron que el FC promedio durante la sesión de entrenamiento fue de 167,64 $\pm 7,05 \mathrm{lpm}$ lo que representa $84,36 \pm 3,52 \%$. El lactato inicial fue de 4,49 $\pm 3,08 \mathrm{mmol} / \mathrm{L}$ yel final 9,23 \pm $3,08 \mathrm{mmol} / \mathrm{L}$ en donde el $\mathrm{p}$-valor para las variables fue de $p=0,0007$. La glucosa inicial fue de 73,6 $\pm 20,71 \mathrm{mg} / \mathrm{dL}$ y la final 76,06 $\pm 20,20 \mathrm{mg} / \mathrm{dL}$ asumiendo un valor de $p=0,7408$. Al tratarse de la composición corporal, el porcentaje

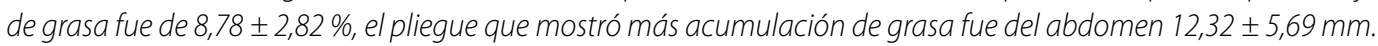
Conclusión: Se concluye que la modalidad parkour tiene predominio del metabolismo anaeróbico glucolítico con el apoyo del metabolismo aeróbico. Además, debido a la especificidad de la modalidad, sus practicantes tienen bajo porcentaje de grasa corporal.

Descriptores: ejercicio, análisis químico de la sangre, metabolismo energético. 


\section{INTRODUÇÃO}

Apesar de possuir pouco tempo no Brasil, a prática do parkour tem se apresentado com grande aceitação e repercussão perante a mídia'. Trata-se de uma prática corporal que visa deslocar-se no ambiente, de um ponto ao outro de forma eficiente, utilizando apenas o corpo ${ }^{2}$. A modalidade surgiu na França e foi disseminada em outros países por meio de documentários, filmes e vídeos na internet ${ }^{1,2}$. No Brasil, chegou em meados de 2004, e desde então tem alcançado destaque na mídia e demais meios de comunicação, chamando atenção de públicos variados.

Devido a tal fato, o número de iniciantes na modalidade tem aumentado. Embora, uma característica muito forte da prática é sua realização na maioria das vezes de forma empírica, acíclica e em ambientes variados, raramente com acompanhamento profissional ${ }^{3}$.

Porém, como se trata de uma modalidade relativamente nova, poucos relatos são apresentados na literatura científica a respeito do parkour, o que faz com que os resultados não sejam conclusivos. Com relação às aptidões motoras, Marchetti et al. ${ }^{4}$ compararam o desempenho muscular e obtiveram resultados superiores em todos os testes, com exceção na força de preensão manual.

No que alude à análise do movimento, Puddle e Maulder ${ }^{5}$, concluíram que as técnicas utilizadas por praticantes reduzem o impacto vertical de forma significativa, podendo ser utilizadas por não praticantes em sua vida cotidiana, e atletas que utilizam saltos durante a prática de suas modalidades.

Para Leite et al. ${ }^{3}$ o parkour pode ser praticado por qualquer pessoa, desde que respeite sua condição física e suas limitações, para os pesquisadores a prática não possui grandes exigências físicas. Porém até o momento nenhum estudo foi encontrando na literatura, que se propôs em investigar as exigências do metabolismo energético e do sistema cardiorrespiratório dos praticantes durante uma sessão de parkour, a fim de determinar a intensidade de esforço.

Entre as variáveis que podem determinar tais efeitos do exercício, destacam-se a frequência cardíaca e os níveis sanguíneos de lactato e glicose, sendo estes últimos, indicadores da via energética utilizada durante uma atividade ${ }^{6}$.

Os dados deste estudo pioneiro servem de apoio para profissionais que trabalham com exercício físico, ensino e treinamento do parkour. Auxiliando na prescrição e melhor orientação da atividade, além de traçar um perfil de alterações que possam ocorrer no organismo e as exigências do metabolismo energético durante a prática.

A pesquisa teve como objetivo geral analisar o comportamento do metabolismo energético e do sistema cardiorrespiratório durante uma sessão de parkour. Como objetivo específico a pesquisa, preocupou-se em determinar os padrões antropométricos dos praticantes de parkour.

\section{MÉTODOS}

O estudo foi caracterizado como quantitativo de caráter transversal, realizado com indivíduos fisicamente ativos praticantes da modalidade parkour. Foram selecionados e participaram da pesquisa, 18 sujeitos com idade média de 22,57 $\pm 2,6$ anos, do sexo masculino, com 4,65 \pm 2,66 anos de prática e uma frequência de treino 2,65 \pm 1,06 vezes por semana, todos da cidade de Curitiba, PR, Brasil. A amostra foi selecionada conforme os seguintes critérios: Inclusão a) indivíduos que tivessem um tempo mínimo de prática de um ano; b) frequência de treino de no mínimo duas vezes por semana. Exclusão: a) indivíduos que no dia da coleta apresentaram algum tipo de lesão osteomioarticular; b) indivíduos que estavam usando intensificadores de desempenho; c) indivíduos que não assinaram e entregaram o Termo de consentimento livre e esclarecido. O estudo foi aprovado pelo Comitê de Ética e Pesquisa da Faculdade Dom Bosco, sob o parecer 146.616, na data 14/11/2012.

Para participar do estudo os sujeitos foram submetidos a alguns procedimentos metodológicos, a saber: antes de iniciar as coletas, foi realizada uma reunião geral a fim de explicar como seriam conduzidas as avaliações; em seguida foi entregue o termo de consentimento livre e esclarecido (TCLE) para que os mesmos assinassem; logo após foram marcadas as avaliações antropométricas e o dia da sessão para realizar o monitoramento da glicose e do lactato a fim de estimar o metabolismo energético predominante durante a sessão de parkour.

A coleta de dados foi realizada por profissionais de Educação Física. Para tanto foram utilizadas as instalações do laboratório de Bioquímica e Fisiologia do Exercício da Faculdade Dom Bosco, Curitiba, Paraná, Brasil. A avaliação antropométrica foi composta por medidas de massa corporal total (MCT), estatura total (ESTT), circunferências, dobras cutâneas tricipital (DCT), subescapular (DCSE), supra-ilíaca (DCSI), peitoral (DCPT), coxa medial (DCCM), abdominal (DCAB) e axilar média (DCAX). (MCT) foi aferida por uma balança digital com estadiômetro (Fabricante Welmy ${ }^{\circledR}$ - Brasil) com capacidade máxima de $300 \mathrm{~kg}$ com subdivisões de $50 \mathrm{~g}$ e estadiômetro com escala de um a dois metros, estando os atletas vestindo roupas leves e descalços, posicionando-se de costas para a plataforma da balança e adotando a posição anatômica (ereto com o olho em um ponto fixo), conforme técnica proposta por $\mathrm{WHO}^{7}$. A (ESTT) foi aferida estando o indivíduo descalço, vestindo roupas leves adotando a posição anatômica (posição ereta, braços estendidos ao longo do corpo) sobre a base da balança. Foi solicitado ao avaliado distribuir a massa corporal igualmente entre ambos os pés, posicionar a cabeça no plano horizontal de Frankfurt, manter os calcanhares unidos, manter os calcanhares, glúteos, escápulas e região occipital em contato com o estadiômetro, realizar uma inspiração profunda e manter apneia para a leitura da medida?

As dobras cutâneas (DCT), (DCSE), (DCAX), (DCSI), (DCPT), (DCCM), (DCAB) foram mensuradas três vezes com um adipômetro científico (Mitutoyo/Cescorf ${ }^{\circledR}$ - Brasil), que exerce uma pressão de $10 \mathrm{~g} / \mathrm{mm}^{2} \mathrm{du}$ rante toda a sua escala de medida $(0,1$ a $88 \mathrm{~mm})$ e com resolução de décimos de milímetros, no lado direito, considerando como resultado final a média aritmética das medidas. A densidade corporal foi estimada pela equação proposta por Jackson e Pollock ${ }^{8}$ desenvolvida originalmente para indivíduos entre 18 e 61 anos e para estimar o percentual de gordura foi utilizada a equação de Siri ${ }^{9}$.

Fórmula de Jackson e Pollock:

\section{$D C=1,11200000-0,00043499\left(\right.$ (27EDC) $+0,00000055(\text { (27EDC })^{2}-$} 0,00028826 (Idade)

Equação de Siri甲 para estimar composição corporal:

$\% \mathrm{G}=[(4,95 / \mathrm{DC})-4,50] \times 100$

onde $\% \mathrm{G}$ é o percentual de gordura corporal.

A avaliação das circunferências foi realizada por meio de uma fita antropométrica (Cescorf - Brasil) com dois metros de comprimento e precisão de $0,1 \mathrm{~cm}$ e foram avaliados os seguintes perímetros: tórax relaxado, tórax inspirado, tórax expirado, cintura, abdômen, quadril, antebraços, braço relaxado, braço contraído, coxa proximal, coxa medial, coxa distal-média e panturrilhas ${ }^{10}$.

A mensuração e o monitoramento da frequência cardíaca foram realizados por meio do frequencímetro da marca Suunto (Finlândia) e do programa (Suunto Monitor). Os avaliados foram monitorados durante uma sessão de parkour que teve uma duração de sessenta minutos, com transmissão em tempo real da frequência cardíaca para um netbook onde estava instalado o programa (Suunto Mananger), programa 
que acompanha o radiofrequencímetro, para que os dados pudessem ser gravados de forma individual. Cabe salientar que o monitoramento começou poucos minutos precedentes ao teste, e só terminou após 10 minutos de recuperação.

As coletas foram realizadas no período da tarde em dois momentos: 30 minutos antes do início da sessão (etapa 1); e logo após o término da sessão (etapa 2); dia 09/03/2013 em Curitiba - PR, no dia a umidade relativa do ar estava em $66 \%$ e a temperatura era de $26^{\circ} \mathrm{C}$.

Para mensurar os determinantes bioquímicos durante a sessão, utilizou-se o aparelho da fabricante (Roche-Suiça), denominado (Accutrend ${ }^{\circledR}$ Plus Lactato), (Accutrend GCT) erro padrão de 0,3111, lancetas estéreis (Accu-Chek - Softclix Pro) caneta lançadora (Accu-Chek - Softclix), tubo capilar de diâmetro interno de um milímetro e sete e meio centímetros de comprimento. Foram utilizados (Accutrend GCT) e (Accutrend ${ }^{\circledR}$ Plus Lactato) já codificados com as fitas correspondentes.

Durante a coleta de sangue, o avaliado estava sentado com a articulação dos braços estendidos ao lado do corpo, e a gota de sangue foi extraída da polpa digital, sendo aplicada sobre área específica da tira reativa e as amostras mensuradas no (Accutrend Lactato) e (Accutrend GCT).

A sessão de parkour foi planejada em um treino misto de percursos e repetições, onde o praticante utilizou as principais técnicas da modalidade, sendo estas: saltos horizontais e verticais, corridas, ultrapassagens de obstáculos e subidas em obstáculos altos. A duração da sessão foi de 60 minutos, sendo 10 minutos destinados ao aquecimento, e o tempo restante às técnicas propriamente ditas.

\section{Análise estatística}

Os dados obtidos foram analisados estatisticamente por meio do programa Microsoft Excel ${ }^{\circledR}$ e apresentados em média, desvio padrão (DP), coeficiente de variação (CV) e amplitude (valores mínimos e máximos).

Para verificar a ocorrência de diferença significativa entre o pré e pós lactato, pré e pós glicose e lado direito e esquerdo dos perímetros, foi realizado o teste $t$-student pareado, assumindo uma significância de $95 \%(p<0,05)$.

\section{RESULTADOS}

A composição corporal dos 18 indivíduos que fizeram parte da amostra é apresentada na Tabela 1. A massa corporal média dos praticantes foi de $(70,3 \pm 6,62 \mathrm{~kg})$, variando entre 58,8 e 88,2 kg. A estatura foi de $(1,75 \pm 0,06 \mathrm{~m})$, variando entre 1,63 e 1,84 metros. A massa magra (MM) dos indivíduos foi de $(64,29 \pm 6,05 \mathrm{~kg})$, variando entre 53,4 e $78,19 \mathrm{~kg}$. A massa gorda (MG) foi de $(6,25 \pm 2,15 \mathrm{~kg})$, variando entre 2,77 e 10,01 kg. O percentual de gordura dos participantes foi de $(8,78 \pm 2,82 \%)$, variando entre 3,92 e 13,59\% demonstrando que a amostra é heterogênea ( $C . V=32 \%)$. Todos os avaliados apresentaram percentual de gordura adequado para o sexo e idade ${ }^{12}$.

A Tabela 2 apresenta os perímetros antropométricos dos 18 dos praticantes de parkour que fizeram parte da amostra. Ao analisar os dados coletados, percebe-se que a amostra é homogênea, não apresentando diferença estatisticamente significativa ( $p \geq 0,05)$, demonstrando que os praticantes de parkour que fizeram parte da amostra eram simétricos.

Tabela 1. Análise da composição corporal de praticantes de Parkour.

\begin{tabular}{c|c|c|c|c|c}
\hline Variáveis antropométricas & Média & D.P & Mínimo & Máximo & CV \\
\hline Massa Corporal (Kg) & 70,3 & 6,62 & 58,8 & 88,2 & $9 \%$ \\
\hline Estatura $(\mathrm{m})$ & 1,75 & 0,06 & 1,63 & 1,84 & $4 \%$ \\
\hline Massa magra (Kg) & 64,29 & 6,05 & 53,4 & 78,19 & $9 \%$ \\
\hline Massa gorda (Kg) & 6,25 & 2,15 & 2,77 & 10,01 & $34 \%$ \\
\hline \% Gordura (\%) & 8,78 & 2,82 & 3,92 & 13,59 & $32 \%$ \\
\hline CV=Coeficiente de variação. D.P.= Desvio Padrão.
\end{tabular}

A Tabela 3 demonstra as dobras cutâneas dos 18 praticantes de parkour avaliados no estudo. Tais dados foram utilizados para a estimativa de percentual de gordura. De acordo com o coeficiente de variação, na maioria das medidas coletadas o grupo mostrou-se como sendo heterogêneo. O ponto de maior acúmulo foi a região abdominal.

O perfil metabólico antes e logo após a sessão de parkour dos 18 praticantes que fizeram parte da amostra é apresentado na tabela 4. A média de lactato de repouso do grupo avaliado foi de $(4,49 \pm 3,08 \mathrm{mMol} / \mathrm{L})$, variando entre 2 e 11,7 mMol/L. Dessa forma, o coeficiente de variação foi de $64 \%$, indicando heterogeneidade do grupo em relação a essa variável. Logo após a sessão de parkour, a média de lactato do grupo avaliado foi de $(9,23 \pm 3,08 \mathrm{mMol} / \mathrm{L})$, variando entre 5,5 e 13,1. Apresentando diferença significativa $(p=0,0007)$. 0 coeficiente de variação de $27 \%$ indica que após a sessão de parkour, o grupo estudado apresentou ser homogêneo.

No que tange os níveis de glicemia antes da sessão de parkour, o grupo apresentou média de $(73,6 \pm 20,71 \mathrm{mg} / \mathrm{dL})$, variando entre 41 e 119 mg/dL. O coeficiente de variação foi de 28\%, demonstrando homogeneidade da amostra. Logo após a sessão de parkour, a média de glicemia aumentou para (76,06 $\pm 20,20 \mathrm{mg} / \mathrm{dL})$, variando entre 44 e 136 mg/dL. O coeficiente de variação de 34\% indica um padrão homogênio da amostra. Os valores não apresentaram diferença significativa $(p=0,7408)$.

Tabela 2. Perímetros antropométricos de praticantes de Parkour.

\begin{tabular}{|c|c|c|c|c|c|}
\hline Perímetros antropométricos $(\mathrm{cm})$ & Média & D.P. & Mínimo & Máximo & CV \\
\hline BD contraído & 33,23 & 3,02 & 27,6 & 39 & $9 \%$ \\
\hline BD relaxado & 30,05 & 3,15 & 24,5 & 36,5 & $10 \%$ \\
\hline$A D$ & 27,09 & 1,48 & 24,3 & 31 & $5 \%$ \\
\hline BE contraído & 33,13 & 3,05 & 27,5 & 39,5 & $9 \%$ \\
\hline BE relaxado & 30,16 & 3,11 & 24,7 & 36,5 & $10 \%$ \\
\hline $\mathrm{AE}$ & 26,5 & 1,38 & 24 & 30 & $5 \%$ \\
\hline Tórax & 93,8 & 5,8 & 83 & 106 & $6 \%$ \\
\hline $\mathrm{TI}$ & 98,98 & 4,76 & 89 & 108 & $5 \%$ \\
\hline TE & 89,7 & 4,87 & 80 & 99 & $5 \%$ \\
\hline Cintura & 75,25 & 5,1 & 68 & 87 & $7 \%$ \\
\hline Abdomen & 80,28 & 6,49 & 70 & 91 & $8 \%$ \\
\hline Quadril & 93,25 & 4,34 & 86,5 & 103 & $5 \%$ \\
\hline CD medial & 53,59 & 3,02 & 48,7 & 62 & $6 \%$ \\
\hline CD proximal & 56,71 & 3,99 & 49,1 & 66 & $7 \%$ \\
\hline CD distal & 47,48 & 3,37 & 43 & 57 & $7 \%$ \\
\hline PD & 36,2 & 1,77 & 35,1 & 39 & $5 \%$ \\
\hline CE medial & 55,3 & 3 & 48 & 61 & $6 \%$ \\
\hline CE proximal & 56,23 & 3,9 & 50 & 66 & $7 \%$ \\
\hline CE distal & 46,89 & 3,13 & 42 & 55 & $7 \%$ \\
\hline PE & 35,94 & 1,72 & 32 & 39 & $5 \%$ \\
\hline
\end{tabular}

$\mathrm{BD}=$ Braço direito; $\mathrm{B}=\mathrm{Braço} \mathrm{esquerdo;} \mathrm{AD}=$ Antebraço direito; $\mathrm{AE}=$ Antebraço esquerdo; $T$ T=Tórax inspirado;

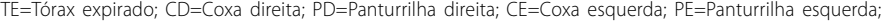
$\mathrm{CV}=$ Coeficiente de variação. D.P.= Desvio Padrão.

Tabela 3. Dobras cutâneas de praticantes de Parkour.

\begin{tabular}{c|c|c|c|c|c}
\hline Dobras cutâneas $(\mathbf{m m})$ & Média & D.P. & Mínimo & Máximo & CV \\
\hline Tríceps & 7,65 & 2,12 & 3,9 & 11,1 & $28 \%$ \\
\hline Subescapular & 10,03 & 2,57 & 6,5 & 16,5 & $26 \%$ \\
\hline Peito & 8,18 & 2,74 & 4 & 12,7 & $34 \%$ \\
\hline Axilar Média & 8,1 & 2,46 & 4,8 & 11,7 & $30 \%$ \\
\hline Suprailíaca & 9,64 & 3,37 & 4,2 & 15,5 & $35 \%$ \\
\hline Abdômen & 12,32 & 5,69 & 5,9 & 25,5 & $46 \%$ \\
\hline Coxa medial & 11,14 & 3,71 & 4,7 & 17,8 & $34 \%$ \\
\hline$\Sigma$ 7DC & 66,03 & 22,69 & 34 & 110,80 & \\
\hline Média $\sum$ DC & 9,58 & 3,72 & 3,9 & 25,5 & \\
\hline
\end{tabular}

$\mathrm{CV}=$ Coeficiente de variação. D.P. $=$ Desvio padrão. $\Sigma 7 \mathrm{DC}=$ Somatório das 7 dobras. $\sum \mathrm{DC}=$ Somatório de dobras cutâneas. 
A Tabela 5 apresenta o perfil da frequência cardíaca (FC) dos 18 praticantes durante a sessão de parkour. A média de FC de repouso foi de (97,27 $\pm 11,78$ bpm), variando entre 80 e 115 bpm. A média da FC durante a sessão de parkour foi de (167,64 $\pm 7,05$ bpm). Percentualmente tais valores representam $(84,36 \pm 3,52 \%)$ da FC máxima de cada indivíduo. A média de FC máxima atingida foi de (197,55 \pm 5,87 bpm), que em percentual representa (98,48 $\pm 1,96 \%$ ) da FC máxima de cada participante.

Tabela 4. Comportamento bioquímico do lactato e glicose antes e logo após uma sessão de Parkour.

\begin{tabular}{c|c|c|c|c}
\hline \multirow{2}{*}{ Análise estatística } & \multicolumn{4}{|c}{ Variáveis Bioquímicas } \\
\cline { 2 - 5 } & \multicolumn{2}{|c|}{ Lactato (mMol/L) } & \multicolumn{2}{c}{ Glicose (mg/dL) } \\
\cline { 2 - 5 } & Repouso & Logo após & Repouso & Logo após \\
\hline Média \pm DP & $4,49 \pm 3,08$ & $9,23 \pm 3,08$ & $73,6 \pm 20,71$ & $76,06 \pm 20,20$ \\
\hline Mínimo & 2 & 5,5 & 41 & 44 \\
\hline Máximo & 11,7 & 13,1 & 119 & 136 \\
\hline CV & $64 \%$ & $27 \%$ & $28 \%$ & $34 \%$ \\
\hline CV=Coeficiente de variação. D.P.= Desvio Padrão.
\end{tabular}

$\mathrm{CV}=$ Coeficiente de variação. D.P.= Desvio Padrão.

Tabela 5. Comportamento da frequência cardíaca durante uma sessão de Parkour.

\begin{tabular}{c|c|c|c|c|c}
\hline Variáveis & FC Repouso & FC média & $\%$ FC & Fc Máx & $\%$ Fc Máx \\
\hline Média \pm DP & $97,27 \pm 11,78$ & $167,64 \pm 7,05$ & $84,36 \pm 3,52$ & $197,55 \pm 5,87$ & $98,48 \pm 1,96$ \\
\hline Mínimo & 80 & 156 & 79,1 & 188 & 95,24 \\
\hline Máximo & 115 & 181 & 90,79 & 206 & 100 \\
\hline CV & $12 \%$ & $4 \%$ & $4 \%$ & $3 \%$ & $2 \%$ \\
\hline
\end{tabular}

$\mathrm{CV}=$ Coeficiente de variação. D.P.= Desvio Padrão; $\mathrm{FC}=$ Frequência cardíaca.

\section{DISCUSSÃO}

As características antropométricas dos praticantes de parkour são apresentadas nas Tabelas 1, 2, 3. As grandes variações quanto à massa corporal 70,3 $\pm 6,62 \mathrm{Kg}$ e estatura 1,75 \pm 0,06 m se devem ao fator de todas as pessoas conseguirem realizar a modalidade. Devido a escassez de informações na literatura sobre a modalidade buscamos informações em esportes e atividades que mais se aproximam de nosso estudo o que torna difícil analisar os dados, devido à falta de indicadores em estudos publicados em periódicos indexados. Devido a essa limitação, os dados foram comparados com modalidades que apresentam características similares como ginástica artística, judô e jiu-jitsu, devido ao forte componente de quedas, rolamentos, destreza ou saltos existente nestas modalidades e no parkour.

Em relação às variáveis antropométricas, estas se apresentam como componente essencial na caracterização de praticantes de qualquer modalidade, pois a composição corporal que é a divisão da massa corporal total em diversos componentes, como massa muscular, massa gorda, massa óssea e massa residual ${ }^{13}$, constitui um dos critérios para a seleção dos indivíduos mais aptos ${ }^{14}$. Segundo Dias et al. ${ }^{15}$ a composição corporal é um componente de importante fator na prevenção de uma série de doenças crônico-degenerativas, e auxilia também na disposição para a realização de tarefas diárias. Os autores reforçam a importância de manter níveis de aptidão física controlados de forma a contribuir para a manutenção da saúde.

No que tange a massa magra (Tabela 1), quanto maior a proporção dessa variável por quilogramas de massa corporal, maior é a força gerada pelo indivíduo ${ }^{13}$. Logo, quantificar esta variável irá permitir analisar o quanto anabólico, para o custo metabólico, estará sendo o programa de treino para o seu praticante, no quesito aumento de força e por consequência melhora da eficiência mecânica ${ }^{16}$. Paoli et al.17, ao estudarem ginastas italianos masculinos relataram valores de massa magra de 64,2 $\pm 6,5 \mathrm{~kg}$ valores que corroboram com nosso estudo. Gómez-Landero et al. ${ }^{18}$, pesquisaram atletas espanhóis masculinos de ginástica de trampolim e encontraram valores para massa magra: músculos 30,69 \pm 4,24 kg; ossos 10,82 \pm 0,84 kg peso residual 15,45 \pm $1,93 \mathrm{~kg}$, valores estes inferiores ao encontrados em nosso estudo. Uma grande quantidade de massa muscular é necessária para aumentar a potência e a força dos movimentos ${ }^{19}$.

Quando comparado com a literatura os valores do percentual de gordura (Tabela 1), foi observado que estes estavam inferiores ao estudo realizado por Grossl et al. ${ }^{20}$, que avaliaram 92 indivíduos com idade de 18 até 29 anos, todos frequentadores de academia e com percentual de gordura de (14,2 $\pm 5,2 \%)$. Andreato et al. ${ }^{14}$, ao avaliar 11 atletas de jiu-jitsu, encontraram valores de 10,3 $\pm 2,6 \%$, valores estes próximos ao nosso estudo. Contudo, ao comparar o referido estudo com atletas de ginástica artística de alto nível ${ }^{17}$, encontraram valores médios de 8,0 \pm $1,3 \%$, valores estes que vem corroborar com a pesquisa. Cabe salientar, percentual de gordura reduzido está correlacionado com um bom nível de treinamento e de suma importância em modalidades na qual o sujeito deve suportar o seu próprio peso corporal ${ }^{19}$.

Ao analisar os dados perimétricos (Tabela 2), observa-se que os praticantes de parkour avaliados neste estudo apresentaram simetria corporal. Tal fato bem provável ocorra por conta da especificidade da modalidade, que utiliza tanto movimentos bilaterais quanto unilaterais ${ }^{5}$. Não havendo assim uma predominância do lado dominante ou não-dominante durante a realização da atividade. Métodos sistematizados de treinamento podem gerar adaptações orgânicas necessárias para o bom desempenho do atleta ${ }^{21}$, porém, modalidades que utilizam movimentos repetitivos com a sobrecarga de treinamento podem causar desequilíbrios musculares acarretando em efeitos deletérios para a postura. Além disso, a repetição errônea de técnicas de execução de movimentos podem acarretar lesões por conta do desequilíbrio muscular ${ }^{22}$.

No que alude as dobras cutâneas (Tabela 3), o somatório é um excelente parâmetro para visualização da gordura subcutânea ${ }^{23}$. Andreato et al. ${ }^{14}$, ao avaliar lutadores de jiu-jitsu encontraram valores de $\Sigma$ 7 DC de 74,4 \pm 18,00 mm, os mesmo autores ao mensurarem a dobra cutânea do abdômen verificaram que esta foi à região que apresentou o maior acúmulo de gordura, média de 15,7 \pm 6,3 mm, valores estes próximos aos encontrados em nosso estudo onde a região abdominal também apresentou os maiores valores. Tal comportamento da gordura na região central é comum no gênero masculino, que possui uma distribuição de gordura no modelo androide, devido principalmente a características hormonais ${ }^{24}$

Ao analisar o comportamento do lactato e glicose (Tabela 4), de acordo com Linnamo et al. ${ }^{25}$, aumentos significativos da concentração de lactato, acima de $10 \mathrm{mMol} / \mathrm{L}$, indicam grande percentual de utilização de fibras rápidas durante o esforço e, portanto, pode ser um bom indicador da potência anaeróbia. Em estudo realizado por Franchini et al. ${ }^{26}$, com atletas de elite de Judô, foram encontradas concentrações pós-luta de 10,08 \pm 2,14 mMol/L, valores estes próximos ao nosso estudo. Pereira et al. ${ }^{27}$, com atletas de jiu-jitsu, encontram valores pós luta de 14,2 \pm 5,9 mMol/L, ilustrando uma grande participação da via glicolítica durante a prática da modalidade.

Níveis de lactato tendem a aumentar rapidamente durante os primeiros minutos de exercício moderado-intenso. Os níveis podem sofrer alterações dependendo das características genéticas, do estado de treinamento do indivíduo, do ambiente, temperatura e desidratação ${ }^{6}$. Uma grande produção de lactato pode nos indicar uma determinada magnitude da capacidade de produção de ATP via metabolismo anaeróbico lático. A formação de lactato ocorre para que a via glicolítica continue funcionando com eficiência, uma vez que esta reação re-oxida a coenzima NAD+27.

Durante a sessão de parkour, faz-se necessária uma manutenção de 
energia para que a atividade consiga ser realizada. Um dos mecanismos dessa manutenção é a utilização da glicose ${ }^{28}$. Durante o exercício ocorre aumento da fosforilação de proteínas relacionadas à captação de glicose pelo músculo esquelético, resultando em uma maior quantidade de GLUT4 transportados para a membrana celular, com consequente aumento na captação de glicose pelo músculo ativo ${ }^{29}$. 0 aumento da glicemia em intensidades supralimiares encontrado neste estudo pode ter ocorrido provavelmente em função da maior atividade adrenérgica, induzindo glicogenólise hepática, bem como por maior atividade gliconeogênica mediada pelo glucagon, uma vez que esses mecanismos de controle ocorram durante exercícios de alta intensidade, podendo explicar a resposta glicêmica ${ }^{30}$.

No que concerne ao comportamento da frequência cardíaca (FC) durante a realização da sessão de parkour (Tabela 5), a FC média apresentou valores de 167,64 \pm 7,05 bpm, valores este que segundo Franchini et al. ${ }^{31}$ indicam que a solicitação cardiovascular durante a sessão não foi tão grande, pois os mesmos autores ao monitorarem a FC durante uma luta de 5 minutos de jiu-jitsu encontraram valores de $158 \pm 14$ bpm, semelhantes ao nosso estudo. O American College of Sports Medicine ${ }^{32}$ recomenda a realização de exercícios físicos de três a cinco vezes por semana, com duração de 20 a 60 minutos contínuos ou intermitentes, intensidade entre moderada e intensa, entre $64 \% \mathrm{e}$ $94 \%$ da frequência cardíaca máxima $\left(F C_{\max }\right.$ ) para a melhora da aptidão cardiorrespiratória. Em nosso estudo a FC média, apresentou-se com $84,36 \pm 3,52 \%$, demonstrando que a sessão de parkour está dentro das recomendações para melhora da aptidão cardiorrespiratória.

\section{CONCLUSÃO}

Com base nos resultados obtidos, observou-se que os praticantes de parkour possuem baixos níveis de percentual de gordura que sugere níveis de massa magra apropriados para uma desejada aptidão física, o que provavelmente proporcionará bons níveis de força e melhora da eficiência mecânica. Em relação ao acúmulo de gordura, a dobra do abdômen foi a que apresentou os maiores valores, devido aos homens possuírem um modelo androide de distribuição de gordura. Analisando o comportamento do lactato, da glicose e da intensidade submáxima da frequência cardíaca, os dados obtidos sugerem que esta modalidade é uma atividade anaeróbia glicolítica, porém com aporte do metabolismo aeróbio. No intuito de um monitoramento metabólico mais eficaz para o parkour, um adendo interessante seria verificar o comportamento dos triglicerídeos e sua demanda metabólica durante a prática da modalidade.

Todos os autores declararam não haver qualquer potencial conflito de interesses referente a este artigo.

CONTRIBUIÇÕES DOS AUTORES: Cada autor contribuiu individual e significantemente para o desenvolvimento deste artigo. GNPS (0000-0002-2979-7282)*, ACF $(0000-0001-7838-062 X)^{*}$ e (0000-0002-2979-7282)* contribuíram nas coletas e redação do manuscrito. MRR (0000-0002-8761-7171)* e JCB (0000-0001-5729-9582)* acompanharam as coletas, orientaram a construção do estudo assim como auxiliaram na análise estatística e revisão. Todos os autores contribuíram de forma intelectual para o estudo. *Número ORCID (Open Researcher and Contributor ID).

\section{REFERÊNCIAS}

1. Atkinson M. Parkour, Anarcho-environmentalism, and poiesis. JSSI. 2009;33(2):169-93.

2. Associação Brasileira de Parkour (ABPK). Manifesto Parkour, 2011. Disponível em: http://www.abpk.org. br/2011/12/manifesto-parkour-abpk/. [Acesso em: 20/abril/2012].

3. Leite N, Aguiar Júnior RP, Cieslak F, Ishiyama M, Milano GE, Stefanello JMF. Perfil de aptidão física dos praticantes de le Parkour. Rev Bras Med Esporte. 2011;17(3):198-201.

4. Marchetti PH, Luz Junior DA, Soares EG, Silva FH, Uchida MC, Teixeira FM. Differences in muscular performance between practitioners and non practitioners of Parkour. Int J Sport Sci. 2012:2(4):36-41.

5. Puddle DL, Maulder PS. Ground reaction forces and loading rates associate with parkour and traditional drop landing techniques. J Sports Sci Med. 2013;12(1):122-9.

6. Lopes RF, Osiecki R, Rama LMPL. Resposta da frequência cardíaca e da concentração de lactato após cada segmento do triathlon olímpico. Rev Bras Med Esporte. 2012:18(3):158-60.

7. World Health Organization. Physical status: the use and interpretation of anthropometry. Report of a WHO Expert Committee. Geneva: WHO; 1995

8. Jackson AS, Pollock ML. Practical assessment of body composition. Phys Sport Med. 1978;13:76-90.

9. Siri WE. Body composition from fluid space and density. In: Brozek J, Henschel A. Techniques for measuring body composition. Washington: National Academy of Science; 1961. p. 223-44.

10. Lohman TG, Roche AF, Martorell R. Anthropometric standardization reference manual. Champaign: Human Kinetics; 1988.

11. Alves JPC, Barbosa MV, Silva RCC, Reis FA, Silva ACS, Santos DN, et al. Estudo comparativo entre analisadores de lactato sanguíneos. Educ Fís Rev. 2012:6(1):1-16.

12. Pollock ML, Wilmore JH. Exercícios na saúde e na doença: avaliação e prescrição para prevenção e reabilitação. São Paulo: Médica e Científica; 1993.

13. Glaner FM, Brito JC. Gordura corporal em judocas: validação cruzada da equação de Lohman. Rev Bras Cineantropom Desempenho Hum. 2007:9(3): 257-61.

14. Andreato LV, Franchini E, Moraes SMF, Esteves JVDC, Pastório JJ, Andreato, TV, et al. Perfil morfológico de atletas de elite de Brazilian Jiu-jitsu. Rev Bras Med Esporte. 2012:18(1):46-50

15. Dias DF, Reis ICB, Reis DA, Cyrino ES, Ohara D, Carvalho FO, et al. Comparação da aptidão física relacionada à saúde de adultos de diferentes faixas etárias. Rev Bras de Cineantropom Desempenho Hum. 2008:10(2):123-128

16. Berral De La Rosa FJ, Rodríguez-Bies EC, Berral De La Rosa CJ, Rojano Ortega D, Lara Padilla E. Comparación de ecuaciones antropométricas para evaluar la masa muscular en jugadores de bádminton. Int. J. Morphol. 2010:28(3): 803-810

17. Paoli A, Grimaldi K, D'Agostino D, Cenci L, Moro T, Bianco A, Palma A. Ketogenic diet does not affect strength performance in elite artistic gymnasts. Int Soc Sports Nutr. 2012;9(1):34.
18. Gómez-Landero LA, Vernetta M, López Bedoya J. Somatotipo y composición corporal en el trampolín masculino español de alto nivel. Rev Intl Cienc Deporte. 2010:19(6):141-53.

19. Deminice R, Rosa TF. Pregas cutâneas vs impedância bioelétrica na avaliação da composição corporal de atletas: uma revisão crítica. Rev Bras Cineantropom Desempenho Hum. 2009:11 (3):334-40.

20. Gross I T, Lima LRA, Karasiak FC. Relação entre a gordura corporal e indicadores antropométricos em adultos frequentadores de academia. Motricidade. 2010:6(2):35-45.

21. Silva LRV. Avaliação da flexibilidade e análise postural em atletas de ginástica rítmica desportiva flexibilidade e postura na ginástica rítmica. Rev Mackenz Educ Físic Esporte. 2008:7(1):59-68.

22. Bastos FN, Pastre CM, Netto Júnior J, Vanderlei LCM, Carvalho Filho G, Hoshi RA, et al. Cor relação entre padrão postural em jovens praticantes do atletismo. Rev Bras Med Esporte. 2009:15(6):432-5.

23. Hyugens W, Claessens AL, Thomis M, Loos R, Van Lagerdonck LV, Peeters M, et al. Body composition: estimations by BIA versus antropometric equations in body builders and other power athletes. J Sports Med Phys Fitness. 2002:42(1):45-55

24. Blouin K, Boivin A, Tchernof A. Androgens and body fat distribution. J Steroid Biochem Mol Biol. 2008;108(3-5):272-80.

25. Linnamo V, Newton RU, Häkkinen K, Komi PV, Davie A, McGuigan M, et al. Neuromuscular responses to explosive and heavy resistance loading. J Electromyogr Kinesiol. 2000;10(6):417-24.

26. Franchini E, Takito MY, Bertuzzi RCM, Kiss MA, Peduti D. Nível competitivo, tipo de recuperação, e remoção do lactato após uma luta de judô. Rev Bras Cineantropom Desempenho Hum. 2004:6(1): 07-16

27. Pereira FR, Lopes RC, Dechechi JC, Victor CB, Ide NB, Navarro CA. Cinética de remoção de lactato em atletas de brazilian jiu-jitsu. RBPFEX. 2011:5(25):39-44.

28. Oliveira ASC, Tibana RA, Aguiar F, Barros E, Silva PB. Relações cineantropométricas e fisiológicas durante exercício incremental em esteira rolante. Rev Bras Med Esporte. 2010:16(4):286-90.

29. Rose AJ, Richter EA. Skeletal muscle glucose uptake during exercise: how is it regulated? Physiology (Bethesda). 2005;20:260-70.

30. Oliveira JC, Baldissera V, Simões HG, Aguiar AP, Azevedo PHSM, Poian PAFO, et al. Identificação do limiar de lactato e limiar glicêmico em exercícios resistidos. Rev Bras Med Esporte. 2006:12(6):333-38.

31. Franchini E, Takito MY, Pereira JNC. Frequência cardíaca e força preensão manual durante a luta de Jiu Jitsu. EFDeportes, Rev Digital Buenos Aires. 2003:9(65). Disponível em: http://www.efdeportes.com/ efd65/jiujitsu.htm. [Aceso em: 20/abril/2012].

32. American College of Sports Medicine. Guidelines for exercise testing and prescription. 7th ed. Baltimore, MD: Lippincott; 2006. 